\title{
Electric signal emissions during repeated abrupt uniaxial compressional stress steps in amphibolite from KTB drilling
}

\author{
D. Triantis ${ }^{1}$, C. Anastasiadis ${ }^{1}$, F. Vallianatos ${ }^{2}$, P. Kyriazis ${ }^{1,3}$, and G. Nover ${ }^{4}$ \\ ${ }^{1}$ Technological Educational Institution of Athens, Athens, Greece \\ ${ }^{2}$ Technological Educational Institute of Crete, Chania, Greece \\ ${ }^{3}$ Brunel University, Uxbridge, London, UK \\ ${ }^{4}$ Mineralogisches Institut der Universität Bonn, Germany
}

Received: 1 December 2006 - Accepted: 4 December 2006 - Published: 26 January 2007

\begin{abstract}
Laboratory experiments have confirmed that the application of uniaxial stress on rock samples is accompanied by the production of weak electric currents, to which the term Pressure Stimulated Currents - PSC has been attributed. In this work the PSC emissions in amphibolite samples from KTB drilling are presented and commented upon. After having applied sequential loading and unloading cycles on the amphibolite samples, it was ascertained that in every new loading cycle after unloading, the emitted PSC exhibits lower peaks. This attitude of the current peaks is consistent with the acoustic emissions phenomena, and in this work is verified for PSC emissions during loading - unloading procedures. Consequently, the evaluation of such signals can help to correlate the state and the remaining strength of the sample with respect to the history of its mechanical stress.
\end{abstract}

\section{Introduction}

In bibliography it has been observed that when mechanical stress is applied upon a rock sample it reacts by emitting electric current (Brady and Rowell, 1986; Enomoto and Hashimoto, 1990; Hadjicontis and Mavromatou, 1994; Takeuchi and Nagahama, 2001; Freund, 2002; Stavrakas et al., 2003). Numerous laboratory experiments including many fracture tests have been conducted on various minerals and rocks both dry and saturated in order to understand and interpret the nature of the mechanisms responsible for the production of such electrical signals (Yoshida et al., 1998; Vallianatos et al., 2004; St-Laurent et al., 2006). The most recent experiment employed an arrangement suitable for recording the electric currents emitted by geomaterial samples during the application of either an abrupt uniaxial stress increase or a monotonically increasing stress up to

Correspondence to: D. Triantis

(triantis@ee.teiath.gr) sample fracture (Triantis et al., 2006). The emitted electric signals were given the name "Pressure Stimulated Currents" (PSC) and the various techniques used to show them up are described by the term "PSC techniques" (Anastasiadis et al., 2004). PSC techniques have been widely used to study the mechanical behaviour of geomaterials and for the investigation of failure mechanisms (Anastasiadis et al., 2004, 2006; Stavrakas et al., 2003).

The present work intends to verify experimentally that pressure stimulated current emissions in amphibolite, produced by loading-unloading procedures that are based on abrupt uniaxial stress variations, show up non-linear response effects as current peaks decrease in each loading cycle and current values are dependent not only on the applied stress, but also on the compression history of the sample. Effects akin to the aforementioned have been mainly studied in detail in acoustic emissions (AE) due to microcrack formation in rocks and other materials during mechanical stress the so-called Kaiser effect, (Kaiser, 1953; Lavrov, 2003, 2005). Kaiser effect in acoustic emissions takes place during reloading of a rock specimen. The first indications of such effects in electric current emissions have been shown up in marble rocks (Stavrakas et al., 2004; Anastasiadis et al., 2007), after the application of three loading - unloading cycles at a constant low stress rate.

\section{Sample characteristics and experimental details}

The material used is a fine grained amphibolite extracted from a depth of the order of $6.0 \mathrm{~km}$ in the drilling site of the German Continental Deep Drilling program (KTB). The main rock forming minerals of the studied amphibolites are hornblende, plagioclase, garnet together with minor quartz and biotite. Accessories included Ti-phases ilmenite 1-3\%, opaques, K-field spar, apatite and some zircon. The poros-

Published by Copernicus GmbH on behalf of the European Geosciences Union. 


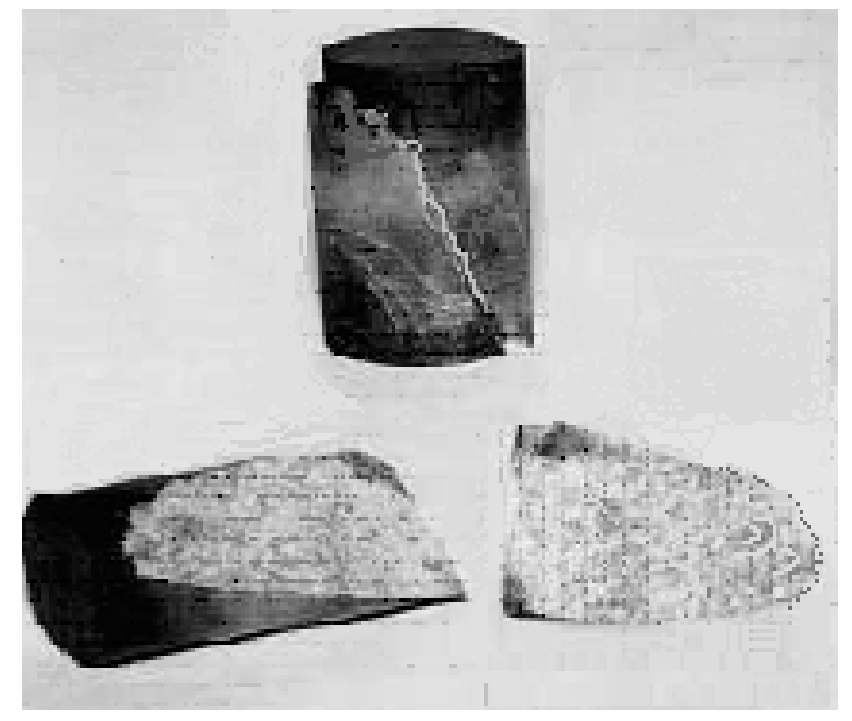

Fig. 1. Photograph of the samples after failure.

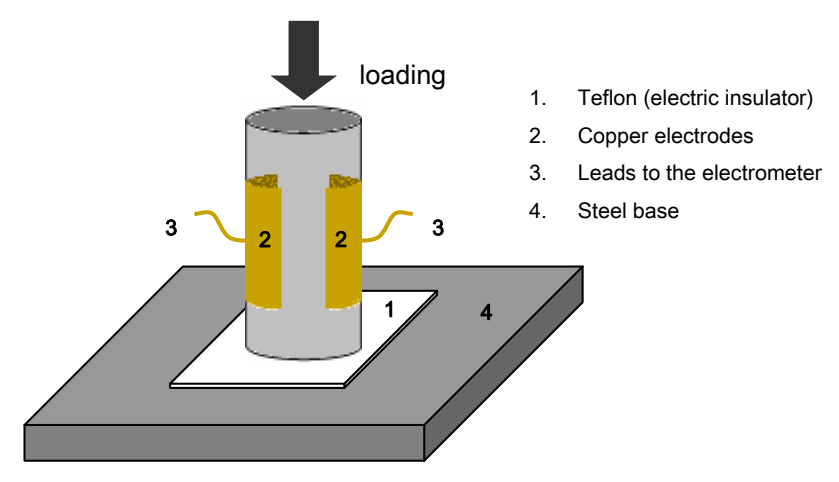

Fig. 2. The experimental setup used for the testing of amphibolite samples.

ity of the samples was less than $1 \%$ vol. (Lich et al., 1992; Nover et al., 1995).

The amphibolite samples used in this experiment were cylindrical measuring $25 \mathrm{~mm}$ in diameter and $50 \mathrm{~mm}$ in height. The average fracture limit was measured to be $85 \mathrm{MPa}$ approximately, after the application of uniaxial compressional stress along their axes. The fracture modes observed were planes diagonal to the direction of stress. The diagonal shearing of the sample is clearly depicted in Fig. 1. The stress-strain curve exhibits an extended linear behaviour up to failure, compared to other rock materials as it is described by Heikamp and Nover (2003).

The experimental technique that was used in this case to show up PSC and to verify the dependence of the accumulated damage on the emitted currents is the so-called StepStress-Technique (SST), whose characteristics have been described by Triantis et al. (2006). More precisely, the sample suffers a constant uniaxial stress $S_{I}$ (initial stress). Then, an (a)

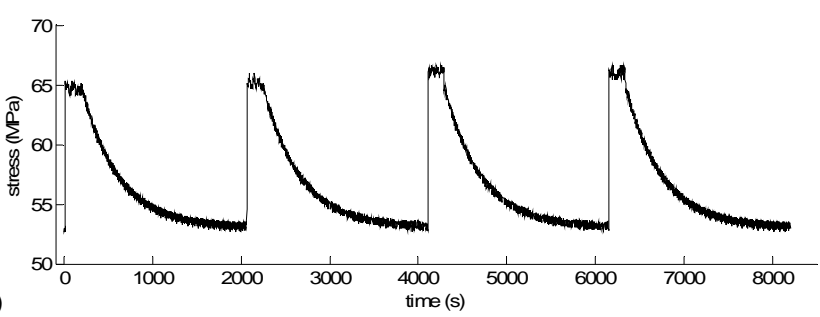

(b)

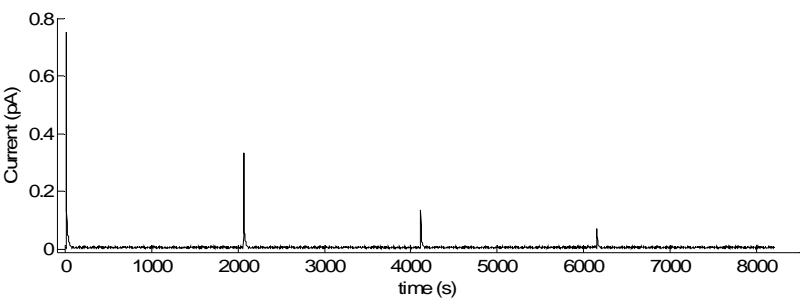

Fig. 3. Presentation of the repeated stress steps (a) and the corresponding PSC recordings with respect to time (b).

abrupt step-wise stress increase $\Delta S=S_{F}-S_{I}$, where $S_{F}$ corresponds to the final level of the applied stress increase, is applied for a short period $\Delta \mathrm{t}$. The stress decreases then at a very slow exponential rate in order to avoid current peaks during unloading and to keep the same zero background current level. The unloading of the sample lasts about $30 \mathrm{~min}$ in each cycle. This procedure is repeated and for each repetition cycle the characteristics of the recorded PSC are investigated.

The experimental setup of the mechanical parts that were used to apply uniaxial compressional stress is depicted in Fig. 2. A pair of oblong copper electrodes measuring $25 \mathrm{~mm}$ in height and $7 \mathrm{~mm}$ in width was attached to the amphibolite sample using conductive paint. The dimensions of the copper electrodes were chosen to have half of the sample height and to be narrow enough, i.e. practically flat when attached to the cylindrical surface of the sample. They were placed opposite to each other with respect to the sample axis, in a direction perpendicular to that of the applied stress (see Fig. 2). Teflon sheets $2 \mathrm{~mm}$ thick were placed on both the upper and the lower surfaces of the sample as electric insulators. In order to perform electrical measurements a sensitive programmable electrometer (Keithley 6514) was used. The experiment was conducted in a Faraday shield to prevent measurements from being affected by electric noise.

\section{Results and discussion}

Four repeated abrupt step-wise axial stress increases were performed in a loading - unloading process within the limits $53 \mathrm{MPa}$ to $65 \mathrm{MPa}$, at identical rates $(4.5 \pm 0.3) \mathrm{MPa} / \mathrm{s}$ (see Fig. 3a), and the corresponding PSC emissions were recorded (see Fig. 3b). Figure 3 presents the time evolution of the whole experiment, while Fig. 4 shows only the first loading step and the corresponding PSC, to outline the de- 
Table 1. Values of the parameters the PSC recording of the four abrupt uniaxial compressional stress step.

\begin{tabular}{lcccc}
\hline Number $n$, the stress step & $n=1$ & $n=2$ & $n=3$ & $n=4$ \\
\hline PSC $_{\max }(\mathrm{pA})$ & 0.75 & 0.34 & 0.14 & 0.07 \\
Total charge Q (pC) & 4.11 & 2.57 & 1.36 & 0.75 \\
PSC peak delay w.r.t. PSC $\max (n=1)$ & 0.0 & 0.8 & 1.5 & 2.2 \\
Slow relaxation time $\tau(\mathrm{s})$ & 22 & 26 & 40 & 67 \\
\hline
\end{tabular}

(a)
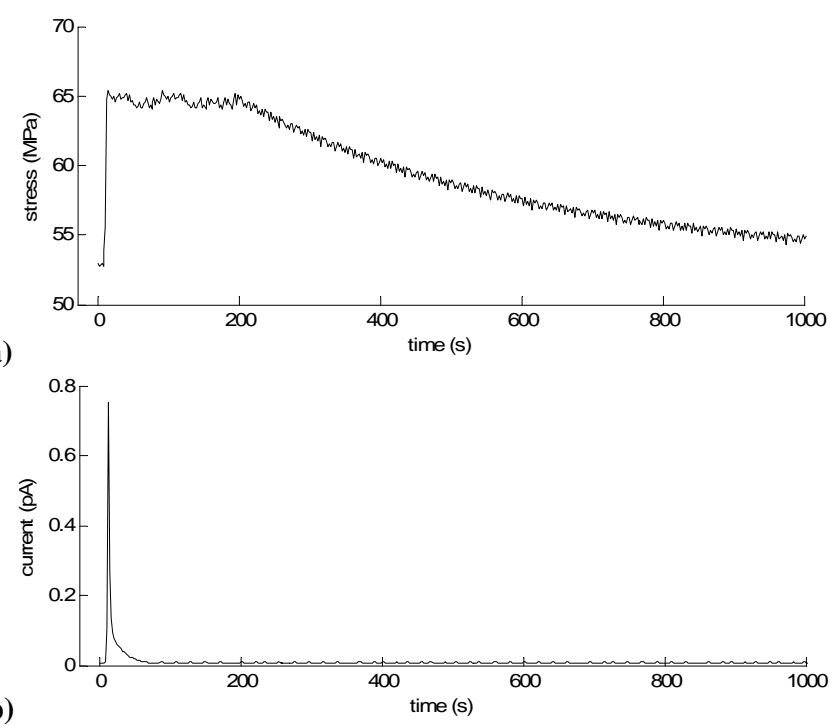

(b)

Fig. 4. Presentation of a single (first) stress step (a) and the corresponding PSC recording with respect to time (b).

tails of the current signal that is related to the abrupt loading. The stress increase rate in each of the four abrupt steps was kept practically constant, in order that the four PSC peak values not to be affected by the different rates (Anastasiadis et al., 2004). Note that a slow unloading scheme was chosen to minimize the unloading effects on current i.e. to avoid probable current spikes of opposite polarity and to keep the same background current values, as abrupt unloading schemes may result in different current relaxation values.

On application of each abrupt stepwise uniaxial compressional stress, an abrupt current spike appears, having a peak at a value $\mathrm{PSC}_{\max }$, as soon as the stress reaches the final level $\mathrm{S}_{F}$. After reaching the PSC peak and while the stress remains at the final level $\mathrm{S}_{F}$, a temporal PSC relaxation down to background level takes place, which is different for each of the four PSC and will be discussed further below.

The decrease of the peak value of $\mathrm{PSC}_{\max }$, in each new loading process up to the final level $\mathrm{S}_{F}$, after a previous unloading to the initial level $S_{I}$, is evident. $\mathrm{PSC}_{\max }$ values for all four abrupt uniaxial compressional stress steps can be read in Table 1.

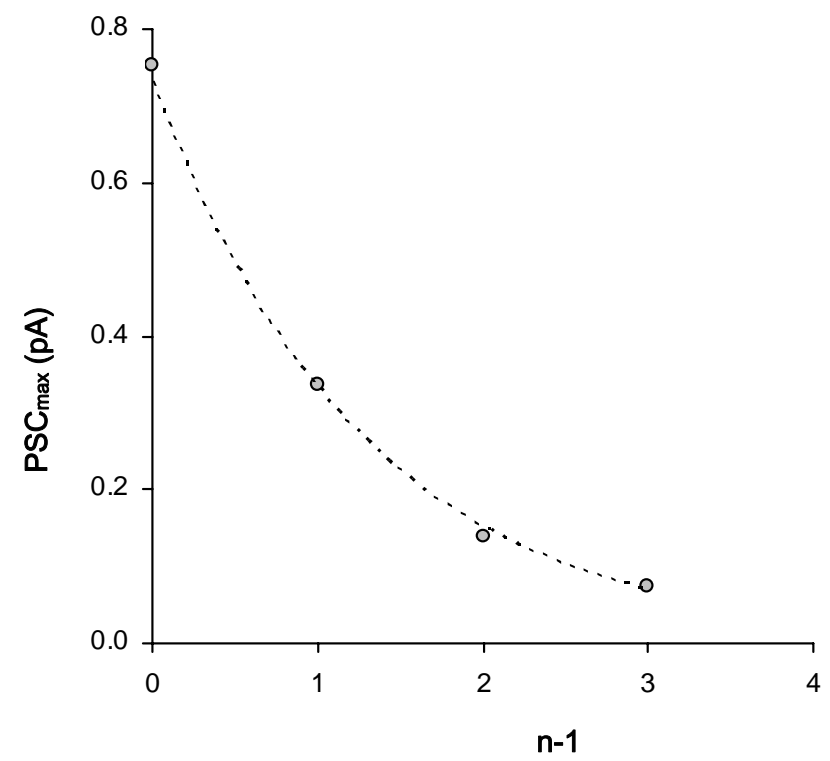

Fig. 5. Decrease rate of PSC peaks for the four successive $(n=4)$ stress steps. The dotted curve is the fitting of the PSC peaks according to Eq. (1).

Figure 5 depicts the experimentally recorded PSC peak $\left(\mathrm{PSC}_{\mathrm{max}}\right)$ exponential decrease rate. After computer-fitting the results, it seems that the depicted curve obeys an exponential law of the form:

$\operatorname{PSC}_{\max }(n)=C \cdot \exp \left(-\frac{n-1}{\lambda}\right), n \in \mathrm{N}$

where $\lambda$ is a factor related to the damage evolution and its value can be used as metric for the damage capacity of a material ( $\lambda$ equals 1.27 for amphibolite). $n$ is the number indicating the loading cycle and $C$ is a factor approaching the value of $\mathrm{PSC}_{\max }$ for $n=1$.

Thus, it was shown experimentally that there is a measurable change in the electric emission behaviour of the material depending on its mechanical stress history, in other words accumulated damage is present and can be shown by means of metrics.

The application of each new compressional stress cycle of the same characteristics causes the removal of the microcrack edges to new excitation positions, resulting in a smaller number of new microcracks, to which the smaller peak values of PSC may be attributed. At the same time, some of the existing dislocations within the material sample are replaced by partially grown neighbouring dislocations corresponding to smaller energy values, so as to meet the requirements of thermodynamics. If no new microcracks were to be formed during each reloading, then, the solid would not suffer fatigue and it would not be aged, so, it would never fail.

It should be noted that a similar expression has been found in the case of marble rocks, after the application of four loading - unloading cycles at a constant low stress rate (Anas- 


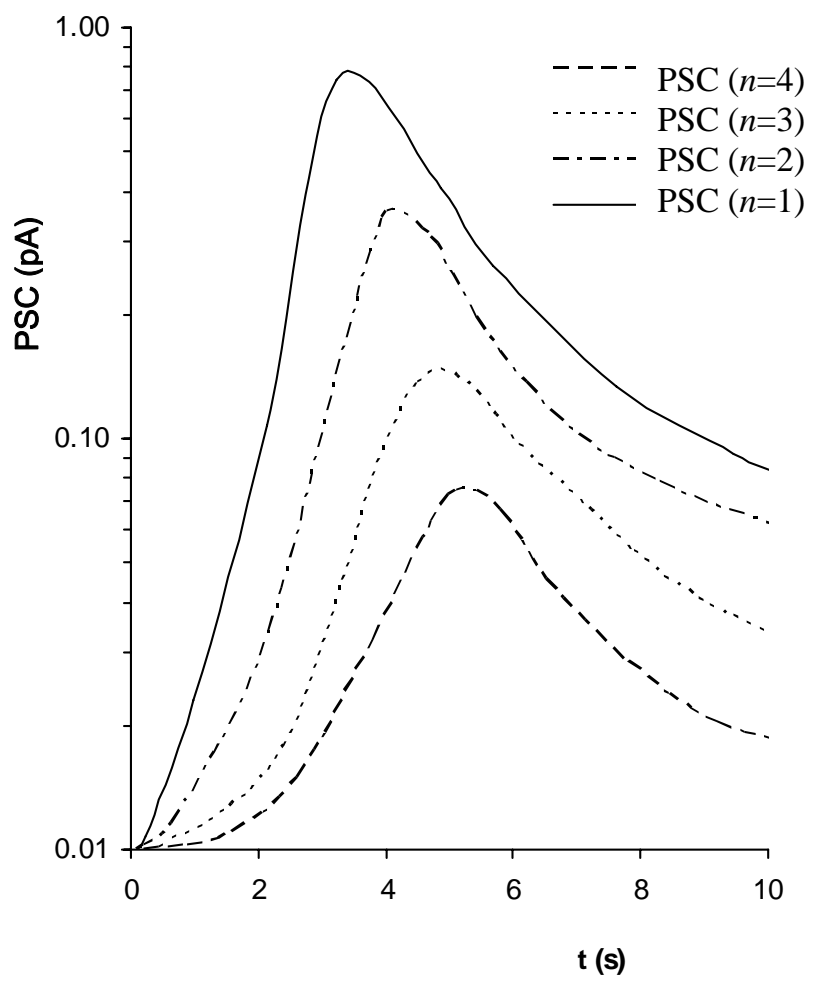

Fig. 6. Comparative presentation of the four PSC peaks (starting point is the moment of the application of stress steps). The horizontal axis is normalized with respect to time.

tasiadis et al., 2007), and the value of factor $\lambda$ was calculated to be equal to 0.92 .

It is not accidental that the mechanical behaviour of amphibolite is characterized by a wider linear range and slightly larger Young's modulus and strength compared to marble (Lama and Vutukuri, 1978). In this work, the amphibolite samples have come from a depth of around $6.0 \mathrm{~km}$ (KTB drilling) and have larger density and grain size distribution (Trčová et al., 2002), thus, clustering of microcracks to macrocracks is less probable. Thus the fracturing of the material is slower in amphibolite than in marble and therefore current peaks can remain high after several loading cycles.

The above facts support the hypothesis that amphibolite can stand more loading cycles and keep PSC emission at measurable levels. This means that new and presumably more microcracks can be produced in amphibolite, before leading the material sample to failure.

The total electric charge released during all four stepwise stress procedures also obeys a decreasing exponential law with respect to time (Eq. 2),

$Q=\int_{0}^{\infty} \operatorname{PSC}(t) \cdot d t$

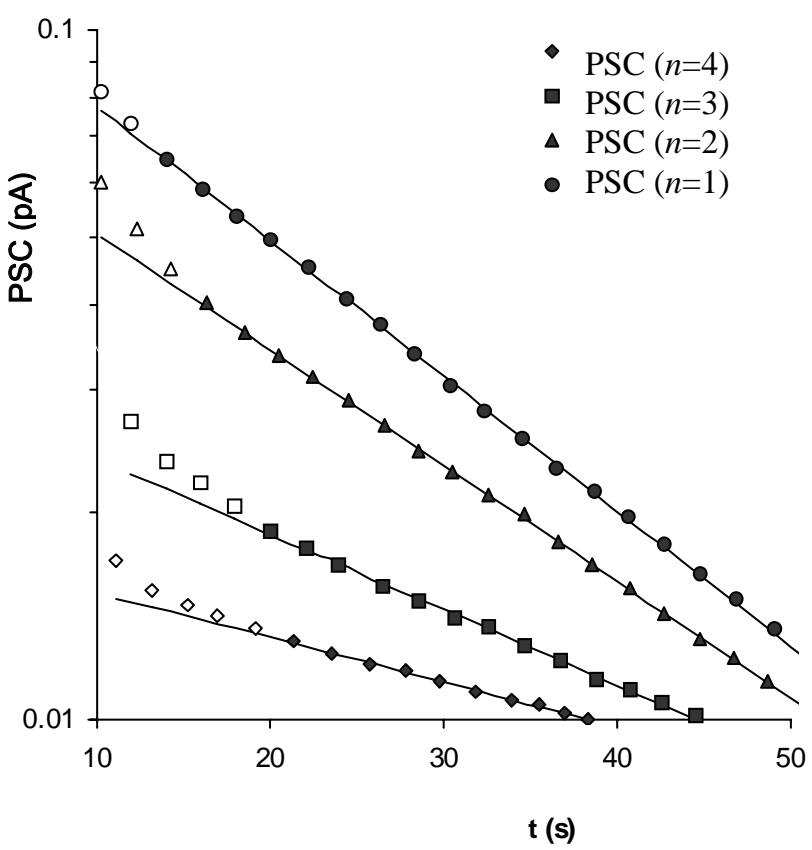

Fig. 7. Slow relaxation mechanism of the four steps. The straight line corresponds to the fitting according to Eq. (2). The open symbols correspond to PSC values deviating from the exponential law of the slow relaxation mechanism.

which is analogous to that described by Eq. (1). Practically the integral goes to $t_{F}$, which is the moment that the value of PSC relaxes to background current i.e. the moment that according to the time constant of the PSC exponential law, the values of PSC are near zero. In Table 1, the total charge values can be read with respect to the four abrupt uniaxial compressional stress steps.

A more analytical and comparative presentation of the four recorded PSC peaks immediately after the application of the stress steps is depicted in Fig. 6. In this diagram the time axis is commonly graduated for the four PSC starting at the moment that the abrupt stepwise stress increase procedure starts while the vertical axis corresponding to PSC values is logarithmic. One may observe a systematic time shift of the PSC peaks as the order $n$ of the stress steps increases. Such a delay in the reaction of the material to the applied stress is expected due to the accumulated damage that is correlated to the stimulus inertia of the material under stress. Notice that the time needed for the stress to reach the final level $S_{F}$ in all four abrupt stepwise stress procedures increase is approximately $3 \mathrm{~s}$. At this moment only the PSC of the first loading cycle has become maximum. The PSC corresponding to the following abrupt stress steps ( $n=2,3$ and 4 ) has a time lag in the appearance of the $\mathrm{PSC}_{\max }$, compared to $\mathrm{PSC}_{\max }$ for $n=1$, that is presented in Table 1.

After the appearance of $\mathrm{PSC}_{\max }$ the current starts relaxing down to background level, at an initially fast rate during 
the first seconds of the relaxation process (see Fig. 6). After maximum and taking into account that the stress remains constant, the microcrack production rate decreases rapidly and consequently the PSC decreases with a short relaxation time. The relaxation of the electric signal, from the mechanical point of view, corresponds to partial closing or - to be more exact - to an infinitesimal approach of the edges of the cracks, which establishes a new equilibrium state. This decrease does not continue at the same rate, because another mechanism may keep the PSC for a long time, so that the PSC relaxation takes place with a longer time constant $\tau$. Consequently, the PSC values for all four abrupt stepwise stress application procedures seem to follow a relaxation process at a fairly slow rate. The experimental results of the PSC recordings show that, for $t \gg t_{m}$, where $t_{m}$ is the instant that the PSC becomes maximum, such a relaxation can be empirically described by the following relation:

$\operatorname{PSC}(t)=A \cdot \exp \left(-\frac{t}{\tau}\right)$

where $A$ is a factor.

Figure 7 depicts the PSC recording of the slow relaxation mechanism of the four abrupt uniaxial compressional stress steps, on a logarithmic current axis with respect to time. Fitting PSC values as a function of time indicates that an exponential relaxation law of the form of Eq. (3) exists. The resulting values for the time constant $\tau$ are given in Table 1. A probable cause of the appearance of the second (slow) relaxation mechanism process is the continuing material strain, even at a very low rate, although stress is unchanged. The new microcracks that go on appearing produce new microcurrents and result in conserving PSC at relatively high values that do not permit a direct relaxation to noise level.

\section{Conclusions}

Phenomena related to the accumulated damage of amphibolite samples that are subjected to uniaxial compressional stress were examined by means of pressure stimulated current emissions caused by loading and unloading of the samples. The experimental results indicate that the electric signals emitted by amphibolite samples during successive loadings and unloadings are characterized by three main features. Firstly, the values of PSC peaks follow an exponential decrease law as the number of loading-unloading cycles increases. A measurable time delay in the occurrence of PSC peaks is present and finally the relaxation time of the PSC increases along with the number of loading-unloading cycles.

All the aforementioned characteristics can serve as criteria of significant importance for testing the ageing and fatigue of the material samples. The results of such experiments verify the existence of accumulated damage effects that associate current emissions with the history of the samples.
Acknowledgements. This work is supported by the project ARCHIMEDES II: "Support of Research Teams of Technological Educational Institute of Athens", sub-project entitled "The electric behavior of geo-materials" in the framework of the Operational Program for Education and Initial Vocational Training. The project is co-funded by the European Social Fund and National Resources.

Edited by: P. F. Biagi

Reviewed by: F. Freund and V. Lapenna

\section{References}

Anastasiadis, C., Triantis, D., Stavrakas, I., and Vallianatos, F.: Pressure stimulated currents (PSC) in marble samples after the application of various stress modes before fracture, Annals of Geophysics, 47, 21-28, 2004.

Anastasiadis, C., Triantis, D., Stavrakas, I., and Vallianatos, F.: Correlation of Pressure Stimulated Currents in rocks with the damage variable, Annals of Geophysics, 49, 6, 2006.

Anastasiadis, C., Triantis, D., and Hogarth, C. A.: Comments on the phenomena underlying pressure stimulated currents in dielectric rock materials, Journal of Materials Science, 10.1007/s10853006-0690-7, in press, 2007.

Brady, B. T. and Rowell G. A.: Laboratory investigation of the electrodynamics of rock fracture, Nature, 321, 488-492, 1986.

Enomoto, J. and Hashimoto H.: Emission of charged particles from indentation fracture of rocks, Nature, 346, 641-643, 1990.

Freund, F.: Charge generation and propagation in rocks, J. Geodyn., 33, 545-572, 2002.

Hadjicontis, V. and Mavromatou, C.: Transient electric signals prior to rock failure under uniaxial compression, Geophys. Res. Lett., 21, 1687-1690, 1994.

Heikamp, S. and Nover, G.: An Integrated study on physical properties of a KTB gneiss sample and marble from Portugal: pressure dependence of the permeability and frequency dependence of the complex electrical impedance, Pure Appl. Geophys., 160, 929-936, 2003.

Kaiser, J.: Erkenntnisse und Folgerungen aus der Messung von Geräuschen bei Zugbeanspruchung von metallischen Werkstoffen, Archiv Eisenhüttenwesen, 24, 43-45, 1953.

Lama, R. D. and Vutukuri, V. S.: Handbook of mechanical properties of rock, vol.2, Trans-Tech, Clausthal, 1978.

Lavrov, A.: The Kaiser effect in rocks: principles and stress estimation techniques, Int. J. Rock Mech. Mining Sci., 40, 151-171, 2003.

Lavrov, A.: Fracture-induced physical phenomena and memory effects in rocks: a review, Strain, 41, 135-149, 2005.

Lich, S., Duyster, J., Godizart, G., Keyssner, S., and de Wall, H.: German Continental Deep Drilling Program (KTB) - Geological survey of the Hauptbohrung 0-6000 m, KTB-Report, 92-2, B1B83, 1992.

Nover, G., Heikamp, A., Kontny, A., and Duba, A.: The effect of Pressure on electrical conductivity of KTB rocks, Surv. Geophys., 16, 63-81, 1995.

Stavrakas, I., Anastasiadis, C., Triantis, D., and Vallianatos, F.: Piezo Stimulated currents in marble samples: Precursory and concurrent with failure signals, Nat. Hazards Earth Syst. Sci., 3, 243-247, 2003, http://www.nat-hazards-earth-syst-sci.net/3/243/2003/. 
Stavrakas, I., Triantis, D., Agioutantis, Z., Maurigiannakis, S., Saltas, V., Vallianatos, F., and Clarke, M.: Pressure stimulated currents in rocks and their correlation with mechanical properties, Nat. Hazards Earth Syst. Sci., 4, 563-567, 2004, http://www.nat-hazards-earth-syst-sci.net/4/563/2004/.

St-Laurent, F., Derr, J., and Freund, F.: Earthquake lights and the stress-activation of positive hole charge carriers in rocks, Phys. Chem. Earth, 31, 305-312, 2006.

Takeuchi, A. and Nagahama, H.: Voltage changes induced by stickslip of granites, Geophys. Res. Lett., 28, 3365-3368, 2001.

Trčová, J., Živor, R., and Přikryl, R.: Physical and Mechanical Properties of Selected amphibolite core samples from Kola Superdeep Borehole KSDB-3, Terra Nova, 14, 379-387, 2002.
Triantis, D., Stavrakas, I., Anastasiadis, C., Kyriazopoulos, A., and Vallianatos, F.: An analysis of pressure stimulated currents (PSC), in marble samples under mechanical stress, Phys. Chem. Earth, 31, 234-239, 2006.

Yoshida, S., Clint, O. C., and Sammonds, P. R.: Electric potential changes prior to shear fracture in dry and saturated rocks, Geophys. Res. Lett., 25, 1577-1580, 1998.

Vallianatos, F., Triantis, D., Tzanis, A., Anastasiadis, C., and Stavrakas, I.: Electric Earthquake Precursors: From Laboratory Results to Field Observations, Phys. Chem. Earth, 29, 339-351, 2004. 